\title{
钴催化共轭烯炔的化学和对映选择性氢化反应
}

\author{
万枫汤文军*
}

(中国科学院上海有机化学研究所 上海 200032)

\section{Cobalt-Catalyzed Chemo- and Enantio-selective Hydrogenation of Conjugated Enynes}

\author{
Wan, Feng Tang, Wenjun* \\ (Shanghai Institute of Organic Chemistry, Chinese Academy of Sciences, Shanghai 200032)
}

过渡金属催化的不对称氢化反应是制备手性化合 物最实用和有效的方法之一. 然而, 迄今为止, 大多数 研究集中于含有单个不饱和键的底物, 对于含有连续多 个不饱和键底物的化学与对映选择性氢化尚未引起关 注. 通常, 在还原反应中 $\mathrm{C} \equiv \mathrm{C}$ 键比 $\mathrm{C}=\mathrm{C} 、 \mathrm{C}=\mathrm{N}$ 和 $\mathrm{C}=\mathrm{O}$ 键具有更高的反应活性, 因此在不对称氢化中, 有效构筑具有 $\mathrm{C} \equiv \mathrm{C}$ 键保持的手性产物具有极大的挑 战. 由于配位活化模式相对接近, 化学和对映选择性地 氢化 $\mathrm{C}=\mathrm{C}$ 键而保留 $\mathrm{C} \equiv \mathrm{C}$ 键则更加困难.

实现烯炔底物选择性氢化的关键因素在于对烯基 和炔基的差异性活化. 在经典的铑一双膦催化体系中, 由于铑较大的原子半径和较强的电子转移能力, 导致其
即使在酰基导向情况下也难以实现 $\mathrm{C} \equiv \mathrm{C}$ 键的保留 ${ }^{[1-2]}$. 上海交通大学化学化工学院张万斌课题组近年来开发 了有效的钴催化不对称氢化体系 ${ }^{[3]}$, 近日又利用具有较 小原子半径和较弱电子转移能力的钴催化体系, 解决了 铑催化体系无法解决的烯基/炔基选择性活化问题，首 次实现了共轭烯炔的高化学和对映选择性氢化 ${ }^{[4]}$.

经过初步的条件优化, 作者发现在 $4.0 \mathrm{Mpa}$ 氢气压 力和 $50{ }^{\circ} \mathrm{C}$ 温度下, 以乙腈作为溶剂, QuinoxP*-CoCl ${ }_{2}-$ $\mathrm{Zn}$ 作为优选催化体系, 对芳环上含有不同位置和电性 取代基的底物均能获得优秀的催化结果, 最终以高化学 选择性和高对映选择性地合成了一系列手性炔丙基胺 类化合物(Scheme 1).

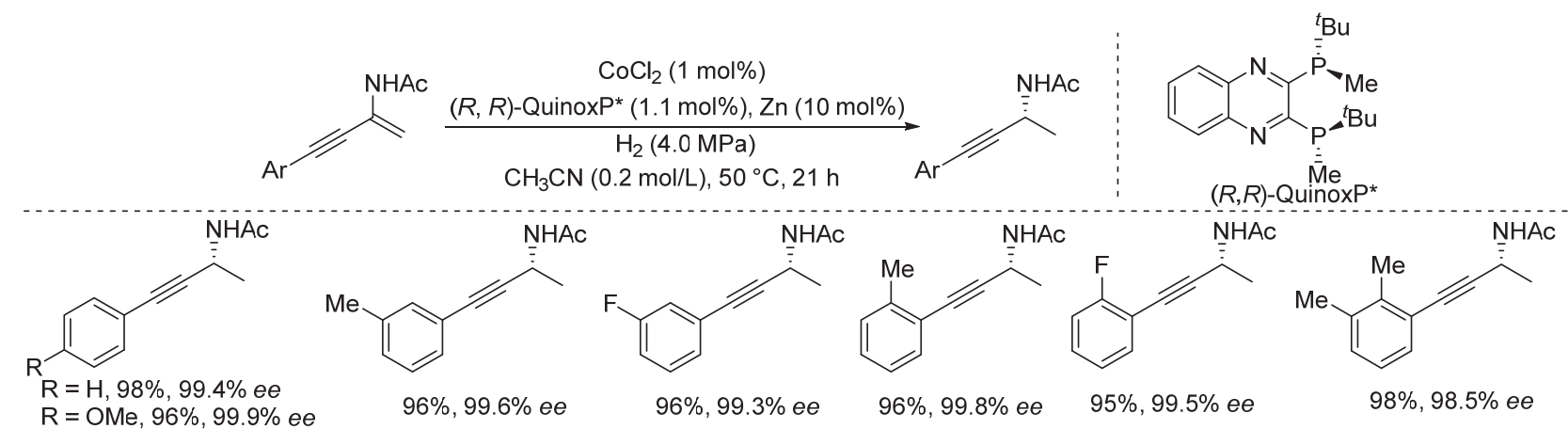

$\mathrm{R}=$ OMe, $96 \%, 99.9 \%$ ee

, $99.6 \%$ ee

$96 \%, 99.3 \%$ ee

$\mathrm{R}=\mathrm{Et}, 95 \%, 99.9 \%$ ee

$\mathrm{R}=n-\operatorname{Pr}, 96 \%, 99.7 \%$ ee

$\mathrm{R}=n-\mathrm{Bu}, 96 \%, 99.8 \%$ ee

$\mathrm{R}=i-\mathrm{Pr}, 94 \%, 99.5 \%$ ee

$\mathrm{R}=t-\mathrm{Bu}, 95 \%, 99.8 \%$ ee

$\mathrm{R}=\mathrm{F}, 95 \%, 99.9 \%$ ee

$\mathrm{R}=\mathrm{Cl}, 97 \%, 99.9 \%$ ee

$\mathrm{R}=\mathrm{CO}_{2} \mathrm{Me}, 94 \%, 99.9 \%$ ee

$\mathrm{R}=\mathrm{CF}_{3}, 97 \%, 99.8 \%$ ee
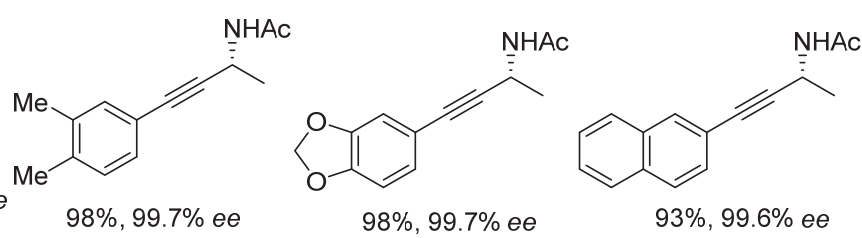

$93 \%, 99.6 \%$ ee
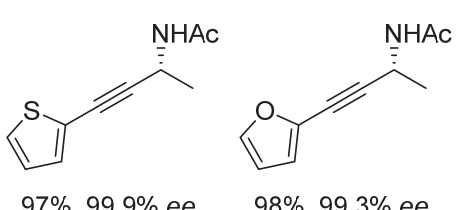

图式 1 底物范围

Scheme 1 Substrate scope

* Corresponding author. E-mail: tangwenjun@sioc.ac.cn. Published online August 11, 2021. 
进一步的研究结果表明, 锌粉还原二价钴络合物随 之生成的锌离子对促进反应起着重要作用. 作者在铑催 化氢化中曾经发现过类似的锌离子促进反应 ${ }^{[5]}$. 他们推 测锌离子通过对氯原子的篗取有利于形成高活性的阳 离子钴中间体, 并提出了可能的 $\mathrm{Co}(\mathrm{I})-\mathrm{Co}(\mathrm{III})$ 氧化还原 反应机理(Scheme 2) ${ }^{[6-7]}$.
该氢化反应可在 $1 / 2000$ 的低催化剂用量下实现 $98 \%$ 的收率和 $98.9 \%$ 的对映选择性(Scheme 3). 氢化产 物通过简单的转化反应可用于多种重要手性化合物(如 乙酰辅酶 $\mathrm{A}$ 羧化酶抑制剂和手性三唑化合物等)的合成 (Schemes 3, 4).

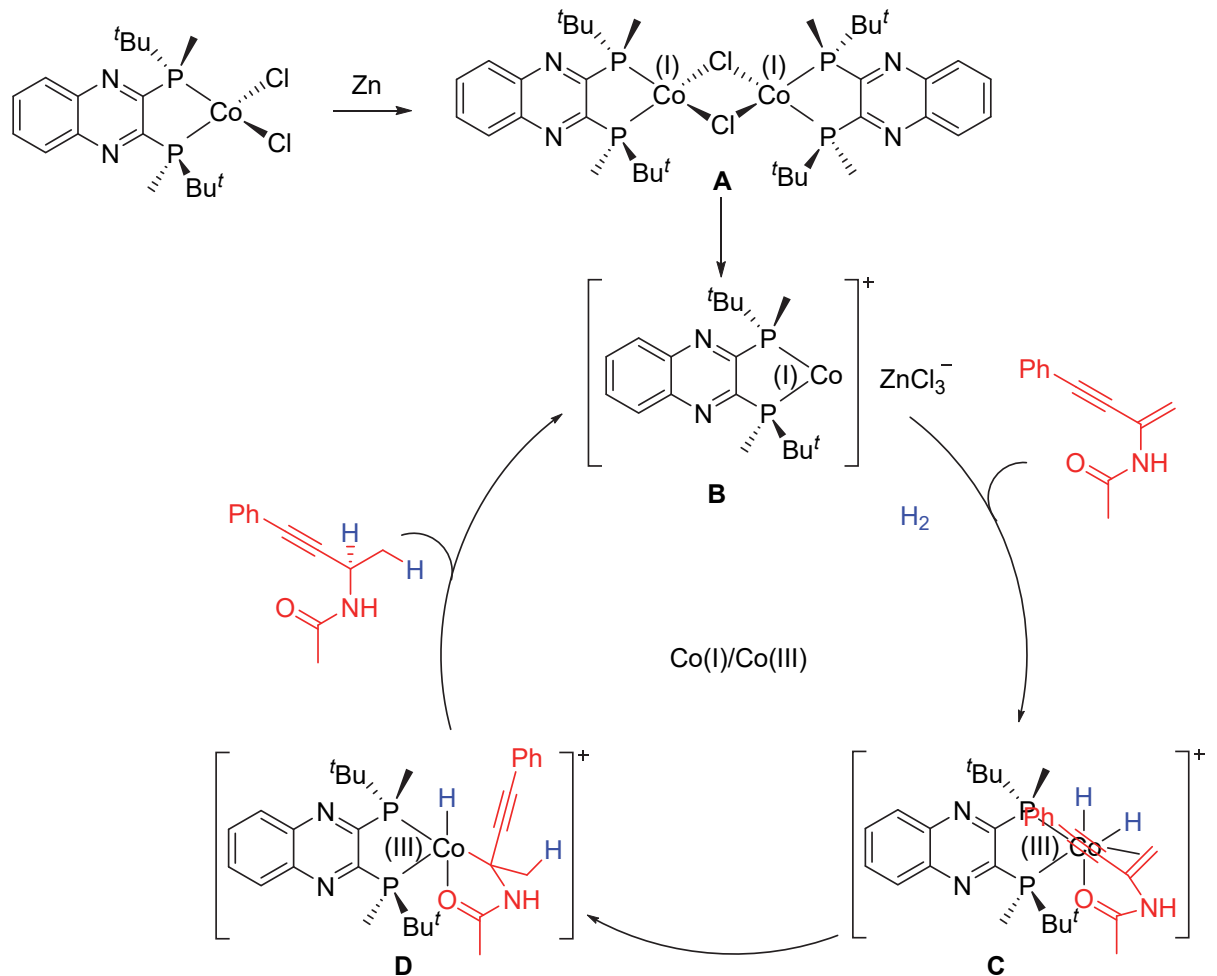

图式 2 可能的 $\mathrm{Co}(\mathrm{I}) / \mathrm{Co}(\mathrm{III})$ 催化循环

Scheme 2 Proposed $\mathrm{Co}(\mathrm{I}) / \mathrm{Co}(\mathrm{III})$ catalytic cycle

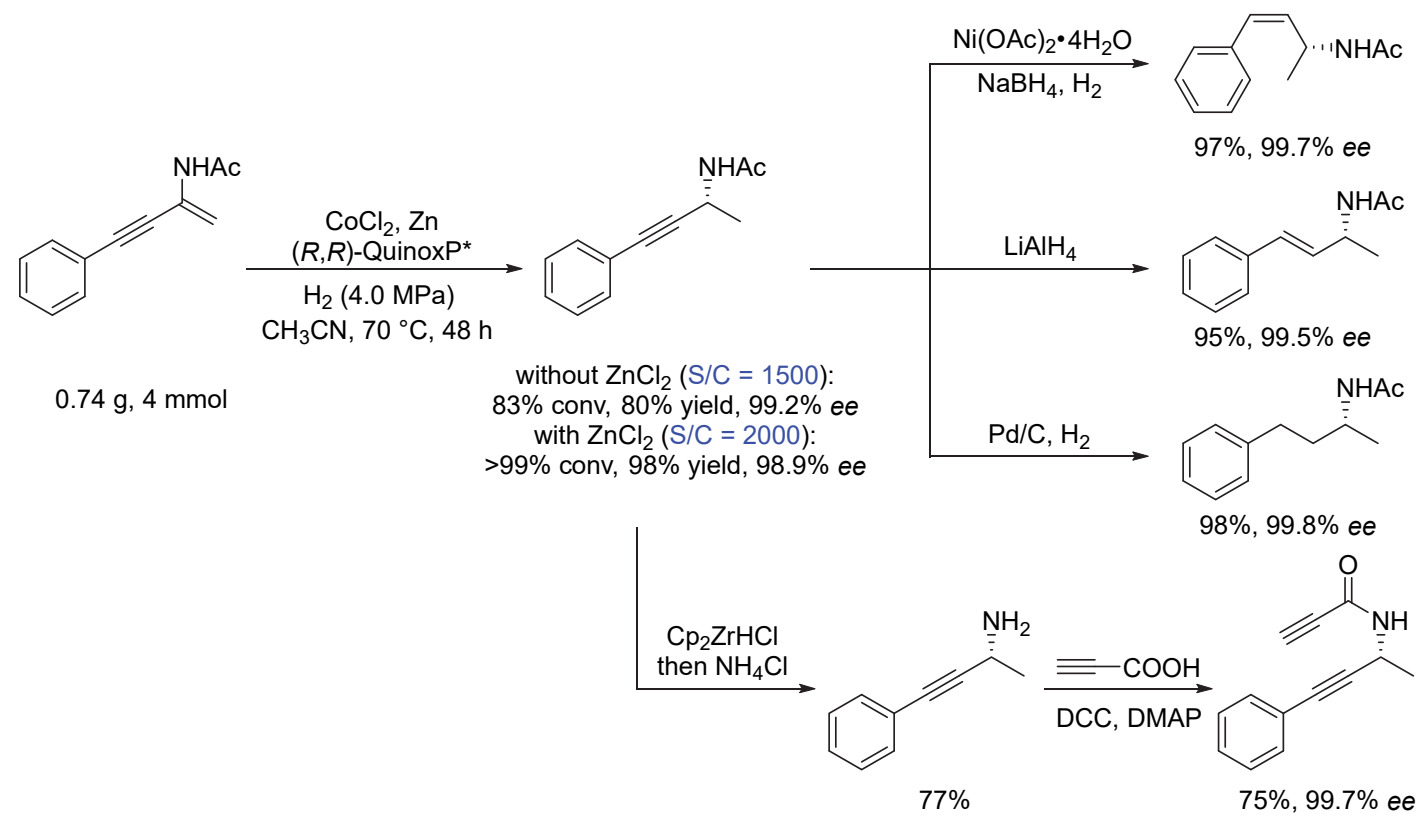

图式 3 转化研究

Scheme 3 Transformation studies 


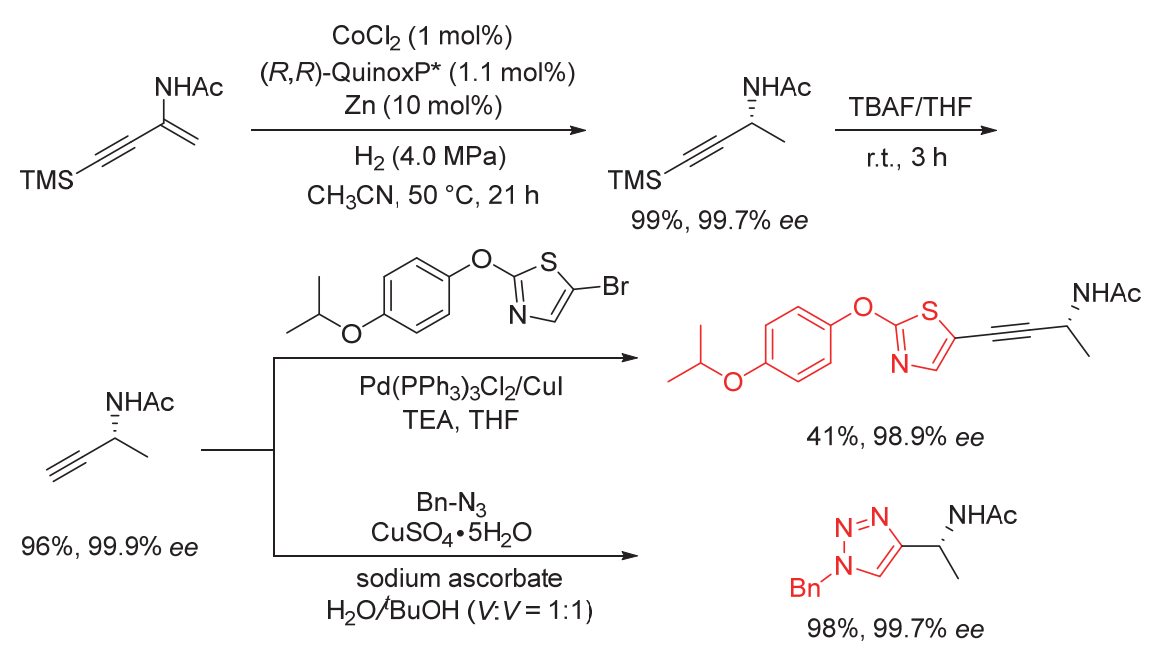

图式 4 应用研究

Scheme 4 Application studies

丰产过渡金属钴催化的不对称氢化反应近年来取 得了不错的进展, 如 Chirik $^{[8] \text { 、陆展 }}{ }^{[9]}$ 、张绪穆 ${ }^{[10]}$ 以及张 万斌 ${ }^{[3]}$ 课题组都报道了开创性的研究成果, 这些催化体 系可以作为铑催化体系在不对称氢化中的补充或替代. 然而, 本文的研究成果实现了过渡金属铑无法实现的共 轭烯炔的选择性不对称氢化, 说明丰产过渡金属除了廉 价易得之外, 在催化性能上也具有区别于稀有过渡金属 的差异化优势.

\section{References}

[1] Liu, T.-L.; Wang, C.-J.; Zhang, X. Angew. Chem., Int. Ed. 2013, 52, 8416.
[2] Boaz, N. W. Tetrahedron Lett. 1998, 39, 5505.

[3] Hu, Y.; Zhang, Z.; Zhang, J.; Liu, Y.; Gridnev, I. D.; Zhang, W. Angew. Chem., Int. Ed. 2019, 58, 15767.

[4] Hu, Y.; Zhang, Z.; Liu, Y.; Zhang, W. Angew. Chem., Int. Ed. 2021, 60, 16989.

[5] Hu, Q.; Zhang, Z.; Liu, Y.; Imamoto, T.; Zhang, W. Angew. Chem., Int. Ed. 2015, 54, 2260.

[6] Ding, W.; Yoshikai, N. Angew. Chem., Int. Ed. 2019, 58, 2500.

[7] Zhong, H.; Friedfeld, M. R.; Chirik, P. J. Angew. Chem., Int. Ed. 2019, 58, 9194.

[8] Friedfeld, M. R.; Shevlin, M.; Hoyt, J. M.; Krska, S. W.; Tudge, M. T.; Chirik, P. J. Science 2013, 342, 1076

[9] Guo, J.; Shen, X.; Lu, Z. Angew. Chem., Int. Ed. 2017, 56, 615

[10] Du, X.; Xiao, Y.; Yang, Y.; Duan, Y.-N.; Li, F.; Hu, Q.; Chung, L. W.; Chen, G.-Q.; Zhang, X. Angew. Chem., Int. Ed. 2021, 60, 11384 .

(Cheng, F.) 